\title{
Avaliação da dissociação iônica do hidróxido de cálcio associado ao Aloe vera como veículo
}

\author{
Evaluation of ionic dissociation of calcium hydroxide associated with Aloe vera as vehicle \\ Maria Carolina de Sousa MELO ${ }^{a}$, Larissa Cordeiro CAVALCANTE ${ }^{a \star}$, Lucas Vaz de OLIVEIRAa, \\ Carmen Milena Rodrigues Siqueira CARVALHO
}

${ }^{a}$ UFPI - Universidade Federal do Piauí, Teresina, PI, Brasil

\begin{abstract}
Resumo
Introdução: O hidróxido de cálcio é considerado uma medicação intracanal padrão ouro e diferentes substâncias podem ser empregadas como veículo para melhorar suas propriedades. O Aloe vera é um fitoterápico popularmente conhecido por babosa e que se destaca pela sua capacidade imunorreguladora, antimicrobiana, cicatrizante e pela biocompatibilidade celular. Objetivo: Avaliar a dissociação iônica do hidróxido de cálcio, tendo o Aloe vera como veículo. Material e método: O extrato de Aloe vera foi obtido diretamente da folha, submetido à filtração e armazenado em tubo Falcon estéril. Foram preparados dois grupos de medicação intracanal: o grupo controle, com hidróxido de cálcio e soro fisiológico, e o grupo experimental, com hidróxido de cálcio e Aloe vera como veículo. Resultado: Não foi verificada uma diferença estatisticamente significativa entre as médias de liberação de cálcio dos grupos $(\mathrm{p}=0,944)$. Quando comparados os valores de forma pareada, considerando as diferentes avaliações ao longo do tempo, foi identificada uma diferença estatisticamente significativa $(\mathrm{p}=0,015)$ para o grupo experimental, de modo que os valores médios da liberação de cálcio foram superiores até o $21^{\circ}$ dia do estudo. Conclusão: $O$ hidróxido de cálcio, quando associado ao Aloe vera, apresentou maior dissociação iônica que quando utilizado com água destilada. Tal resultado, associado às propriedades inerentes deste fitoterápico, sugerem que a pasta de hidróxido de cálcio e Aloe vera pode ser uma alternativa viável e promissora como medicação intracanal, e melhorar o reparo apical e periapical.
\end{abstract}

Descritores: Aloe vera; endodontia; hidróxido de cálcio.

\begin{abstract}
Introduction: Calcium hydroxide is considered an intracanal medication gold standard, and different substances can be employed as a vehicle to improve its properties. Aloe vera is a herbal remedy popularly known as babosa and is notable for its immunoregulatory, antimicrobial, healing and cellular biocompatibility. Objective: To evaluate the ionic dissociation of calcium hydroxide, with the Aloe vera as vehicle. Material and method: The extract of Aloe vera was obtained directly from the leaf, submitted to filtration and stored in a sterile falcon tube. Two groups of intracanal medication were prepared; the control group, with calcium hydroxide and saline; and the experimental group, with calcium hydroxide and Aloe vera as vehicle. Result: There was no statistically significant difference between the means of calcium release from the groups $(\mathrm{p}=0.944)$. When comparing the paired values, considering the different evaluations over time, a statistically significant difference $(\mathrm{p}=0.015)$ was identified for the experimental group, so that mean values of calcium released were higher up to the 21st day of the study. Conclusion: Calcium hydroxide, when associated with Aloe vera, presented higher ionic dissociation than when used with distilled water. This result, combined with the inherent properties of this herbal remedy, suggests that the paste of calcium hydroxide and Aloe vera can be a viable and promising alternative as intracanal medication and improve apical and periapical repair.
\end{abstract}

Descriptors: Aloe vera; endodontics; calcium hydroxide.

\section{INTRODUÇÃO}

As medicações intracanais são utilizadas como coadjuvantes no tratamento endodôntico, com o intuito de reduzir as bactérias remanescentes e seus metabólitos, e acelerar o processo de cicatrização ${ }^{1,2}$. O hidróxido de cálcio $\left(\mathrm{Ca}(\mathrm{OH})_{2}\right)$ é a substância mais utilizada com essa finalidade. Seu mecanismo de ação é resultado da dissociação de íons hidroxila e cálcio, com consequente aumento de $\mathrm{pH}$, o que lhe confere ação antimicrobiana e indutora de mineralização, com possibilidade de reparo dos tecidos periapicais adjacentes ${ }^{3-7}$.

Diferentes substâncias podem ser empregadas como veículo para o $\mathrm{Ca}(\mathrm{OH})_{2}$, as quais podem melhorar ou modificar suas 
propriedades $^{4,8}$. Soro fisiológico, paramonoclorofenol, glicerina, anestésico e clorexidina são exemplos de substâncias utilizadas como coadjuvantes à ação do $\mathrm{Ca}(\mathrm{OH})_{2}^{7,9}$.

No intuito de melhorar a pasta utilizada como medicação intracanal, o Aloe vera (AV), membro da família das Liliáceas, tem sido avaliado como veículo para o hidróxido de cálcio ${ }^{4}$. Popularmente conhecido como babosa, esse fitoterápico se destaca pela sua capacidade imunorreguladora, antimicrobiana, cicatrizante e pela biocompatibilidade celular ${ }^{10-17}$

Dessa forma - e posto que a atividade de reparo tecidual e a ação antimicrobiana são fatores importantes no sucesso do tratamento endodôntico -, o objetivo deste estudo foi avaliar a dissociação iônica do hidróxido de cálcio, tendo Aloe vera como veículo.

\section{METODOLOGIA}

\section{Obtenção e Preparo do Extrato de Aloe vera}

O Aloe vera (Aloe barbadensis Miller) foi cultivado no Núcleo de Pesquisas em Plantas Medicinais, Centro de Ciências Agrárias, da Universidade Federal do Piauí. A fim de manter a homogeneidade das amostras utilizadas, as folhas foram obtidas do mesmo cultivo e sob as mesmas condições de umidade e temperatura.

Para a obtenção do extrato, as folhas foram cortadas a $5 \mathrm{~cm}$ do talo, lavadas em água corrente e colocadas em um recipiente com água destilada, por 24 horas, com a finalidade de evitar a contaminação do gel com a seiva no momento da extração. Em seguida, as folhas foram desinfetadas com etanol a 70\%. Sob condições estéreis, o gel de Aloe vera foi removido por raspagem da folha, com espátula n. ${ }^{\circ} 24$, homogeneizado e filtrado em Filtro Disco PureFlo de $25 \mathrm{~mm}$, micragem de 0,45 $\mu \mathrm{m}$ (ZenPure Corporation, São Paulo, Brasil), e armazenado em tubo Falcon estéril, sem exposição à luz ${ }^{16}$. O extrato final foi conservado em temperatura média de 7 graus Celsius.

\section{Análise}

A análise foi processada no Laboratório de Química da Universidade Federal do Piauí. As substâncias utilizadas para a pesquisa foram o hidróxido de cálcio P.A. (Biodinâmica, São Paulo, Brasil), o extrato de Aloe vera, o soro fisiológico (Cloreto de sódio 0,9\% - Indústria Farmacêutica Ltda. HALEXLSTAR, Goiás, Brasil) e a água destilada (Asfer, São Caetano do Sul, São Paulo, Brasil).

As pastas de hidróxido de cálcio (Biodinâmica, São Paulo, Brasil) foram padronizadas de acordo com a proporção vide bula, em quantidade necessária para a análise:

- Grupo 1 (controle): $1 \mathrm{~g}$ de hidróxido de cálcio P.A.; $10 \mathrm{~mL}$ soro fisiológico.

- Grupo 2 (experimental): 1 g de hidróxido de cálcio P.A.; $10 \mathrm{~mL}$ extrato da planta Aloe vera.

A mensuração do hidróxido de cálcio foi realizada em balança eletrônica analítica (SHIMADZU, Barueri, São Paulo, Brasil). A porção líquida foi mensurada em pipeta (KASVI, Curitiba, Paraná,
Brasil). Após a homogeneização, cada grupo foi acondicionado em copo de Becker e armazenado a $7^{\circ} \mathrm{C}$.

O estudo foi realizado em triplicata. A concentração de cálcio livre foi mensurada através de um espectrofotômetro digital modelo SP1105 (Bel Photonics, São Paulo, Brasil), com o auxílio de reagentes Calcio Liquiform (Labtest, Minas Gerais, Brasil). As mensurações foram feitas em sete tempos: imediato, após 1 hora, 24 horas, 7 dias, 14 dias, 21 dias e 28 dias.

Os valores foram calculados de acordo com o preconizado pelos reagentes Calcio Liquiform (Labtest, Minas Gerais, Brasil). Para o cálculo da concentração de cálcio livre (mg/L), utilizou-se:

$$
\mathrm{Ca}\left(\frac{\mathrm{mg}}{\mathrm{L}}\right)=\frac{\mathrm{A}_{\text {amostra }}}{\mathrm{A}_{\text {padrão }}} \times 100
$$

\section{Estatística}

Os dados do estudo foram organizados em planilha do Microsoft Excel $^{\circledR}$ e foram processados no software IBM ${ }^{\circledR}$ SPSS $^{\circledR}$, versão 21.0 . Foram calculadas estatísticas descritivas, como médias, desvio padrão, mínimos e máximos, para as variáveis quantitativas.

Foi utilizado o Teste de Shapiro-Wilk para verificação da normalidade das distribuições. Foi realizado o Teste t independente de Student para comparação das médias de liberação de cálcio dos grupos. Foi utilizado o Teste t de Student para amostras pareadas, para comparação das diferentes avaliações de liberação de cálcio ao longo do tempo, entre os grupos. Todas as análises foram realizadas no nível de significância de 5\%.

\section{RESULTADO}

Os resultados das concentrações de cálcio livre nos dois grupos podem ser observados nas Tabelas 1 e 2, a seguir, de acordo com cada tempo. A Tabela 1 mostra os valores (absorbância e concentração de cálcio livre) de cada grupo em triplicata, os desvios padrão e relativo. A partir da mensuração no sétimo dia, o desvio padrão aumentou para os dois grupos. Por fim, houve um pequeno aumento das concentrações médias com 28 dias e diminuição do desvio padrão.

Não foi verificada uma diferença estatisticamente significativa entre as médias de liberação de cálcio dos grupos $(\mathrm{p}=0,944)$, de modo global, embora o grupo que recebeu extrato de Aloe vera tenha obtido uma média (DP) de 144,7 $(17,3)$ e o grupo que recebeu soro fisiológico 144,1 $(16,9)$ (Tabela 3). Quando comparados os valores de forma pareada, considerando-se as diferentes avaliações ao longo do tempo, foi identificada uma diferença estatisticamente significativa $(p=0,015)$ para o grupo 2 , de modo que os valores médios da liberação de cálcio foram superiores até o 21. ${ }^{\circ}$ dia, com uma diferença variando de $0,12 \mathrm{mg} / \mathrm{L}$ a $1,47 \mathrm{mg} / \mathrm{L}$. O valor do $28 .^{\circ}$ dia foi o único inferior, com diferença de $0,07 \mathrm{mg} / \mathrm{L}$.

$\mathrm{Na}$ análise gráfica (Figura 1), os dois grupos tiveram comportamentos semelhantes, com concentração de cálcio livre ( $\mathrm{mg} / \mathrm{L})$ diminuindo ao longo do tempo, sendo essa diminuição mais evidente no intervalo de zero a sete dias. 
Tabela 1. Absorbância das amostras, média aritmética, desvio padrão e desvio padrão relativo da concentração de cálcio livre dos grupos 1 e 2

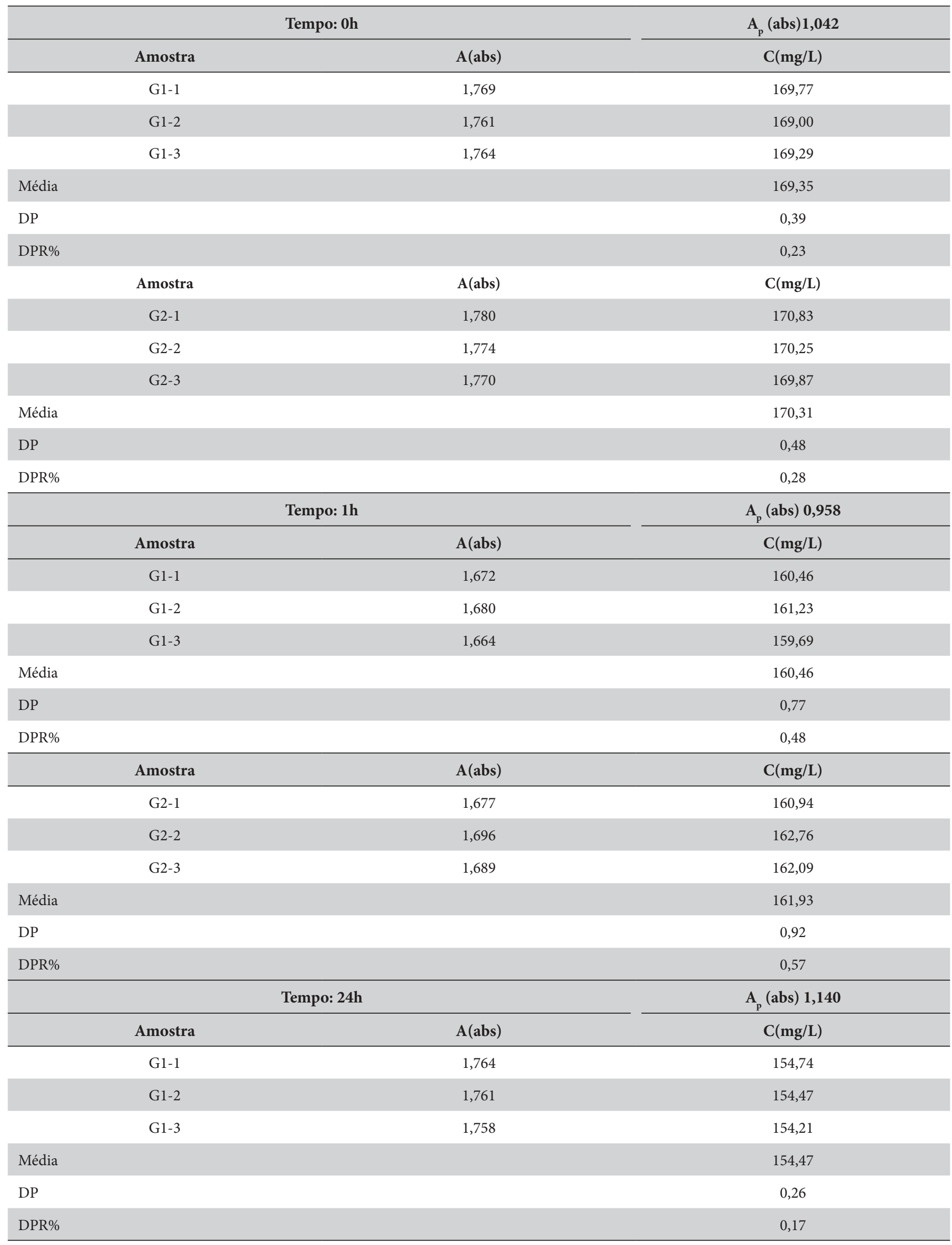


Tabela 1. Continuação...

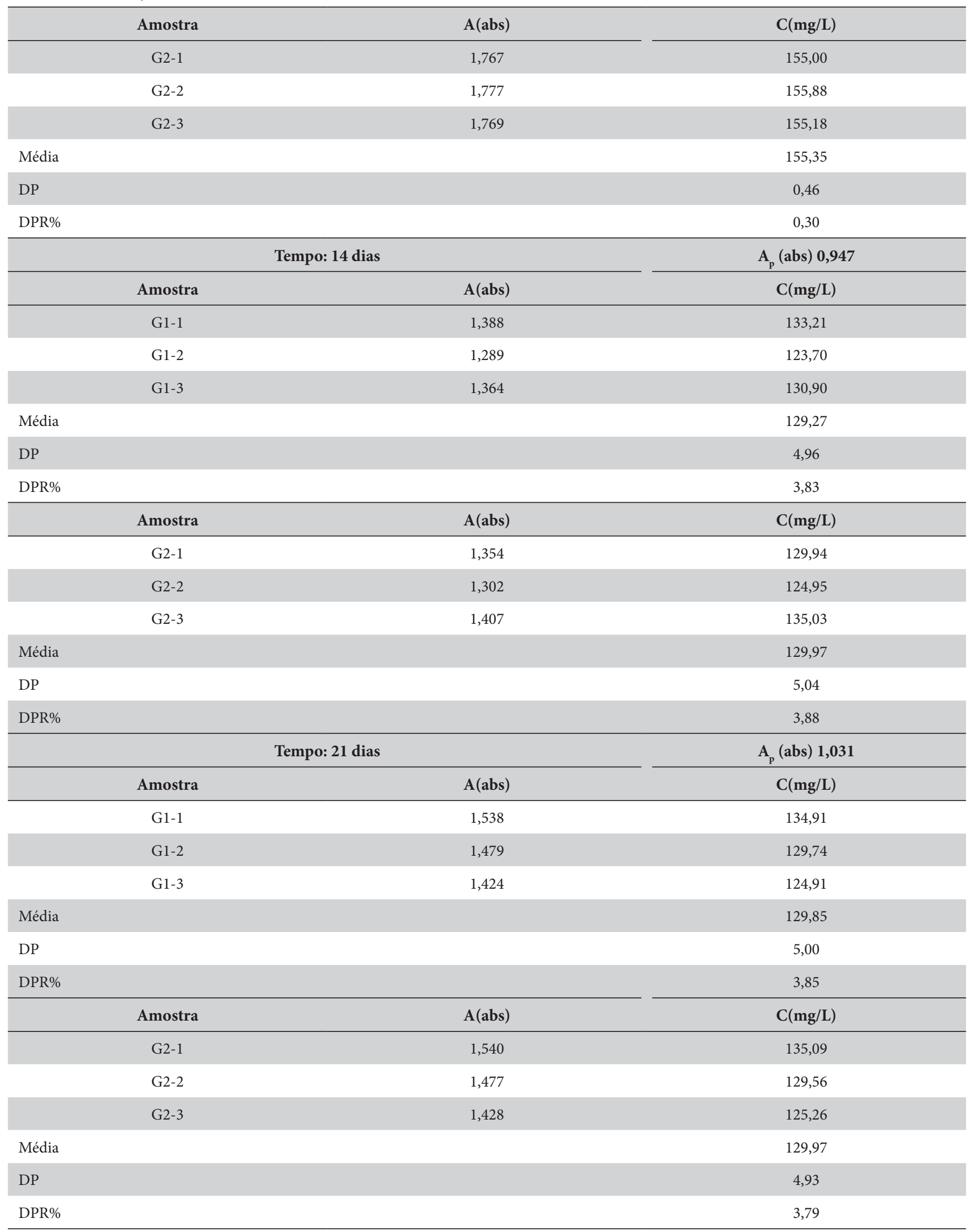


Tabela 1. Continuação...

\begin{tabular}{|c|c|c|}
\hline \multicolumn{2}{|c|}{ Tempo: 28 dias } & \multirow{2}{*}{$\frac{A_{p}(a b s) 0,993}{C(m g / L)}$} \\
\hline Amostra & A(abs) & \\
\hline G1-1 & 1,402 & 134,55 \\
\hline G1-2 & 1,369 & 131,38 \\
\hline G1-3 & 1,309 & 125,62 \\
\hline Média & & 130,52 \\
\hline $\mathrm{DP}$ & & 4,52 \\
\hline DPR\% & & 3,47 \\
\hline Amostra & $A(a b s)$ & $\mathrm{C}(\mathrm{mg} / \mathrm{L})$ \\
\hline G2-1 & 1,315 & 126,20 \\
\hline G2-2 & 1,362 & 130,71 \\
\hline $\mathrm{G} 2-3$ & 1,401 & 134,45 \\
\hline Média & & 130,45 \\
\hline DP & & 4,13 \\
\hline DPR\% & & 3,17 \\
\hline
\end{tabular}

Tabela 2. Valores médios da liberação de cálcio (mg/L) para os grupos G1 e G2 em 0h, 1h, 24h, e 7, 14, 21 e 28 dias após a mistura

\begin{tabular}{|c|c|c|}
\hline \multirow{3}{*}{ Tempos } & Grupo 1 & Grupo 2 \\
\hline & Hidróxido de cálcio & Hidróxido de cálcio \\
\hline & Soro fisiológico & Extrato de Aloe Vera \\
\hline $\mathbf{O h}$ & 169,35 & 170,31 \\
\hline $1 \mathrm{~h}$ & 160,46 & 161,93 \\
\hline $24 \mathrm{~h}$ & 154,47 & 155,35 \\
\hline $7 d$ & 134,56 & 135,12 \\
\hline $14 d$ & 129,27 & 129,97 \\
\hline 21d & 129,85 & 129,97 \\
\hline $28 d$ & 130,52 & 130,45 \\
\hline
\end{tabular}

Tabela 3. Análise estatística global

\begin{tabular}{|c|c|c|c|c|c|c|c|c|c|c|}
\hline & & \multicolumn{2}{|c|}{$\begin{array}{c}\text { Teste de Levene } \\
\text { para igualdade das } \\
\text { variâncias }\end{array}$} & \multicolumn{7}{|c|}{ Teste - T para comparação de médias } \\
\hline & & \multirow[t]{2}{*}{$\mathbf{F}$} & \multirow[t]{2}{*}{ Sig. } & \multirow[t]{2}{*}{$\mathbf{t}$} & \multirow[t]{2}{*}{ df } & \multirow[t]{2}{*}{ Sig. } & \multirow{2}{*}{$\begin{array}{l}\text { Diferença } \\
\text { média }\end{array}$} & \multirow{2}{*}{$\begin{array}{l}\text { Diferença } \\
\text { de erro } \\
\text { padrão }\end{array}$} & \multicolumn{2}{|c|}{$\begin{array}{c}95 \% \text { intervalo de } \\
\text { confiança da diferença }\end{array}$} \\
\hline & & & & & & & & & inferior & superior \\
\hline \multirow{2}{*}{$\begin{array}{l}\text { Lib } \\
\text { Cálcio }\end{array}$} & $\begin{array}{l}\text { Variâncias } \\
\text { homogêneas }\end{array}$ & 019 &, 894 &,- 072 & 12 & ,944 &,- 66000 & 9,13667 & $-20,567$ & 19,2471 \\
\hline & $\begin{array}{c}\text { Variâncias } \\
\text { não } \\
\text { homogêneas }\end{array}$ & & &,- 072 & 11,993 & ,944 &,- 66000 & 9,13667 & $-20,568$ & 19,24836 \\
\hline
\end{tabular}


Tabela 4. Análise estatística pareada

\begin{tabular}{|c|c|c|c|c|c|c|c|c|c|}
\hline & & \multicolumn{5}{|c|}{ Diferenças pareadas } & \multirow{3}{*}{$\mathbf{T}$} & \multirow{3}{*}{ Df } & \multirow{3}{*}{ Sig. } \\
\hline & & \multirow{2}{*}{ Média } & \multirow{2}{*}{$\begin{array}{l}\text { Desvio } \\
\text { padrão }\end{array}$} & \multirow{2}{*}{$\begin{array}{l}\text { Média de } \\
\text { erro padrão }\end{array}$} & \multicolumn{2}{|c|}{$\begin{array}{c}\text { 95\% intervalo de confiança } \\
\text { da diferença }\end{array}$} & & & \\
\hline & & & & & Inferior & superior & & & \\
\hline Par 1 & $\begin{array}{l}\text { Grupo_1 } \\
\text { Grupo_2 }\end{array}$ &,- 6600 & ,52131 & ,19704 & $-1,14213$ &,- 17787 & $-3,350$ & 6 &, 015 \\
\hline
\end{tabular}

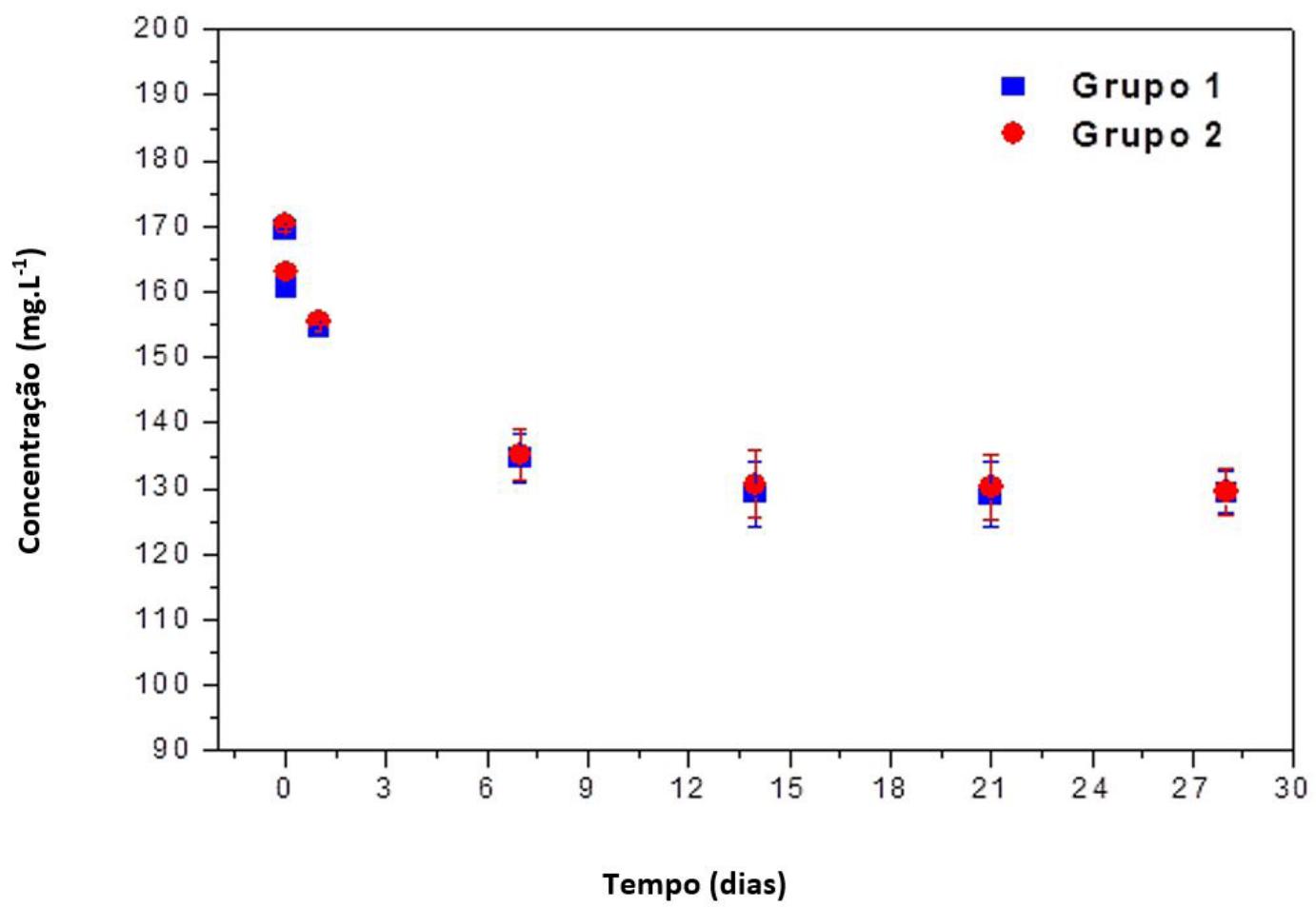

Figura 1. Concentração de cálcio livre (mg/L), de acordo com o tempo.

\section{DISCUSSÃO}

As propriedades induzidas pelo hidróxido de cálcio $\left(\mathrm{Ca}(\mathrm{OH})_{2}\right)$ são decorrentes do seu mecanismo de dissociação iônica, com liberação de íons hidroxila e consequente aumento do $\mathrm{pH}$. Dentre suas principais características, destacam-se a atividade antimicrobiana, a limitação da reabsorção radicular, a indução de tecido mineralizado e a promoção do reparo tecidual periapical, com ação anti-inflamatória secundária ${ }^{9,18-20}$.

Diferentes veículos são adicionados ao hidróxido de cálcio, através da formação de pastas. A efetividade dessas substâncias é consequência da influência que promovem na dissociação iônica e na capacidade de difusão do $\left(\mathrm{Ca}(\mathrm{OH})_{2}\right)$ entre os túbulos dentinários, suas ramificações e o forame apical, interferindo nas propriedades da pasta sobre os canais ${ }^{4,9}$. Além disso, o veículo pode influenciar na solubilidade e na capacidade de reabsorção da pasta pelos tecidos periapicais ${ }^{3}$.

Os veículos aquosos, devido à rápida promoção de dissociação iônica, apresentam ampla indicação clínica. O soro fisiológico e a água destilada são substâncias comprovadamente efetivas como veículos para o hidróxido de cálcio ${ }^{3,21}$. Com base nisso, o soro fisiológico foi utilizado como grupo controle para a execução deste estudo.

Batista et al. ${ }^{4}$ avaliaram, com o auxílio de pHmetro, pastas contendo Aloe vera (AV) em dentes bovinos e concluíram que o fitoterápico possibilitou a dissociação iônica do $\mathrm{CaOH}_{2}$ através dos túbulos dentinários. Além disso, evidenciaram a necessidade de novas pesquisas sobre o tema.

De fato, a análise em espectrofotômetro realizada neste estudo observou dados semelhantes aos relatados anteriormente. O AV, além de permitir a dissociação iônica do $\mathrm{Ca}(\mathrm{OH})_{2}$, não demonstrou alterações estatisticamente significativas quando comparado ao soro fisiológico (Tabelas 1, 2 e 3).

No entanto, a análise isolada de cada um dos tempos da pesquisa (Tabelas 2 e 4) demonstrou que a dissociação iônica de $\mathrm{Ca}(\mathrm{OH})_{2}$ no grupo experimental foi superior em relação ao grupo controle, até o $21 .^{\circ}$ dia. Isso sugere que o Aloe vera pode induzir positivamente a dissociação dessa medicação intracanal, quando comparado a veículos já consolidados na literatura, como o soro fisiológico. 
A capacidade de dissociação iônica, aliada às propriedades específicas desse fitoterápico, sugere que o AV pode ser um eficaz veículo para o hidróxido de cálcio quando utilizado como medicação intracanal.

A utilização de Aloe vera em contato direto com fibroblastos de polpa humana ${ }^{18}$, ou mesmo como meio de armazenamento de dentes avulsionados ou quebrados ${ }^{16}$, confirma sua biocompatibilidade com células pulpares e baixa citotoxicidade ${ }^{18}$.

Além disso, destaca-se seu potencial cicatrizador e anti-inflamatório, que pode ser um coadjuvante no tratamento de patologias endodônticas ${ }^{14,22}$ quando aliado a outras medicações intracanais. Sua utilização como veículo para o Mineral Trióxido Agregado (MTA), por exemplo, demonstrou redução significativa dos efeitos da cascata inflamatória em experimentos in vivo ${ }^{12}$.

O potencial cicatrizador do Aloe vera é decorrente do efeito imunomodulador de polissacarídeos presentes na planta, que regulam a expressão de mediadores inflamatórios ${ }^{23,24}$. O Acemannan é o principal polissacarídeo extraído do gel de Aloe vera e pode promover a proliferação celular de polpa dentária e diferenciação e mineralização da matriz extracelular ${ }^{25}$. As propriedades anti-inflamatórias desta planta estimulam o crescimento dos fibroblastos, o que acelera o processo de cicatrização e reparação tecidual ${ }^{18}$.

\section{CONCLUSÃO}

O hidróxido de cálcio, quando associado ao Aloe vera, apresentou maior dissociação iônica que quando utilizado com água destilada. Tal resultado, associado às propriedades inerentes deste fitoterápico, sugere que a pasta de hidróxido de cálcio e Aloe vera pode ser uma alternativa viável e promissora como medicação intracanal, e melhorar o reparo apical e periapical.

\section{AGRADECIMENTOS}

Os autores agradecem à Universidade Federal do Piauí UFPI, pelo apoio logístico para a execução do trabalho.

\section{REFERÊNCIAS}

1. Rezende GC, Massunari L, Queiroz IOA, Gomes JE Fo, Jacinto RC, Lodi CS, et al. Antimicrobial action of calcium hydroxide-based endodontic sealers after setting, against E. faecalis biofilm. Braz Oral Res. 2016;30(1):e38. http://dx.doi.org/10.1590/1807-3107BOR-2016.vol30.0038. PMid:26981759.

2. de Pablo OV, Estevez R, Heilborn C, Cohenca N. Root anatomy and canal configuration of the permanent mandibular first molar: clinical implications and recommendations. Quintessence Int. 2012 Jan;43(1):15-27. PMid:22259805.

3. Mohammadi Z, Dummer PMH. Properties and applications of calcium hydroxide in endodontics and dental traumatology. Int Endod J. 2011 Aug;44(8):697-730. http://dx.doi.org/10.1111/j.1365-2591.2011.01886.x. PMid:21535021.

4. Batista VE, Olian DD, Mori GG. Diffusion of hydroxyl ions from calcium hydroxide and Aloe vera pastes. Braz Dent J. 2014;25(3):212-6. http://dx.doi.org/10.1590/0103-6440201300021. PMid:25252256.

5. Saatchi M, Shokraneh A, Navaei H, Maracy MR, Shojaei H. Antibacterial effect of calcium hydroxide combined with chlorhexidine on Enterococcus faecalis : a systematic review and meta-analysis. J Appl Oral Sci. 2014 Sep-Oct;22(5):356-65. http://dx.doi.org/10.1590/1678775720140032. PMid:25466470.

6. Abbaszadegan A, Sahebi S, Gholami A, Delroba A, Kiani A, Iraji A, et al. Time-dependent antibacterial effects of Aloe vera and Zataria multiflora plant essential oils compared to calcium hydroxide in teeth infected with Enterococcus faecalis. J Investig Clin Dent. 2016 Feb;7(1):93-101. http://dx.doi.org/10.1111/jicd.12123. PMid:25187255.

7. Carvalho CN, Freire LG, Carvalho APL, Duarte MAH, Bauer J, Gavini G. Ions release and pH of calcium hydroxide-, chlorhexidine- and bioactive glass-based endodontic medicaments. Braz Dent J. 2016 Jun;27(3):325-31. http://dx.doi.org/10.1590/0103-6440201600602. PMid:27224568.

8. Zmener O, Pameijer CH, Banegas G. An in vitro study of the $\mathrm{pH}$ of three calcium hydroxide dressing materials. Dent Traumatol. 2007 Feb;23(1):21-5. http://dx.doi.org/10.1111/j.1600-9657.2005.00447.x. PMid:17227376.

9. Vianna ME, Zilio DM, Ferraz CCR, Zaia AA, Souza-Filho FJ, Gomes BPFA. Concentration of hydrogen ions in several calcium hydroxide pastes over different periods of time. Braz Dent J. 2009;20(5):382-8. http://dx.doi.org/10.1590/S0103-64402009000500005. PMid:20126906.

10. Costa CR, Amorim BR, Magalhães P, Canto GL, Acevedo AC, Guerra EN. Effects of plants on osteogenic differentiation and mineralization of periodontal ligament cells: a systematic review. Phytother Res. 2016 Apr;30(4):519-31. http://dx.doi.org/10.1002/ptr.5568. PMid:26822584.

11. Fani M, Kohanteb J. Inhibitory activity of Aloe vera gel on some clinically isolated cariogenic and periodontopathic bacteria. J Oral Sci. 2012 Mar;54(1):15-21. http://dx.doi.org/10.2334/josnusd.54.15. PMid:22466882.

12. Fé JLM, Coelho CA, Damascena GM, Soares IMV, Alves FR, Santos IMSP, et al. Aloe vera as vehicle to mineral trioxide aggregate: study in bone repair. Rev Odontol UNESP. 2014 Oct;43(5):299-304. http://dx.doi.org/10.1590/rou.2014.048.

13. Jittapiromsak N, Sahawat D, Banlunara W, Sangvanich P, Thunyakitpisal P. Acemannan, an extracted product from Aloe vera, stimulates dental pulp cell proliferation, differentiation, mineralization, and dentin formation. Tissue Eng Part A. 2010 Jun;16(6):1997-2006. http:// dx.doi.org/10.1089/ten.tea.2009.0593. PMid:20088703.

14. Maenthaisong R, Chaiyakunapruk N, Niruntraporn S, Kongkaew C. The efficacy of Aloe vera used for burn wound healing : a systematic review. Burns. 2007 Sep;33(6):713-8. http://dx.doi.org/10.1016/j.burns.2006.10.384. PMid:17499928. 
15. Salazar-Sánchez N, López-Jornet P, Camacho-Alonso F, Sánchez-Siles M. Efficacy of topical Aloe vera in patients with oral lichen planus : a randomized double-blind study. J Oral Pathol Med. 2010 Nov;39(10):735-40. http://dx.doi.org/10.1111/j.1600-0714.2010.00947.x. PMid:20923446.

16. Sholehvar F, Mehrabani D, Yaghmaei P, Vahdati A. The effect of Aloe vera gel on viability of dental pulp stem cells. Dent Traumatol. 2016 Oct;32(5):390-6. http://dx.doi.org/10.1111/edt.12272. PMid:27126516.

17. Sudarshan R, Annigeri RG, Vijayabala GS. Aloe vera in the treatment for oral submucous fibrosis - a preliminary study. J Oral Pathol Med. 2012 Nov;41(10):755-61. http://dx.doi.org/10.1111/j.1600-0714.2012.01168.x. PMid:22650317.

18. Carvalho NC, Guedes SAG, Albuquerque-Júnior RLC, Albuquerque DS, Araújo AAS, Paranhos LR, et al. Analysis of Aloe vera cytotoxicity and genotoxicity associated with endodontic medication and laser photobiomodulation. J Photochem Photobiol B. 2018 Jan;178:348-54. http://dx.doi.org/10.1016/j.jphotobiol.2017.11.027. PMid:29178996.

19. Duarte M, Marchi AO, Giaxa M, Kuga M, Fraga SC, Souza L. Evaluation of pH and calcium ion release of three root canal sealers. J Endod. 2000 Jul;26(7):389-90. http://dx.doi.org/10.1097/00004770-200007000-00002. PMid:11199761.

20. Han GY, Park SH, Yoon TC. Antimicrobial activity of $\mathrm{Ca}(\mathrm{OH}) 2$ containing pastes with Enterococcus faecalis in vitro. J Endod. 2001 May;27(5):328-32. http://dx.doi.org/10.1097/00004770-200105000-00004. PMid:11485250.

21. Athanassiadis B, Abbott PV, Walsh LJ. The use of calcium hydroxide, antibiotics and biocides as antimicrobial medicaments in endodontics. Aust Dent J. 2007 Mar;52(1 Suppl):S64-82. http://dx.doi.org/10.1111/j.1834-7819.2007.tb00527.x. PMid:17546863.

22. Nimma VL, Talla HV, Bairi JK, Gopaldas M, Bathula H, Vangdoth S. Holistic healing through herbs: effectiveness of aloe vera on post extraction socket healing. J Clin Diagn Res. 2017 Mar;11(3):ZC83-6. PMid:28511517.

23. Devaraj A, Karpagam T. Evaluation of anti-inflammatory activity of and analgesic effect of Aloe vera leaf extract in rats. Int Res J Pharm. 2011 Mar;2(3):103-10.

24. Esua MF, Rauwald JW. Novel bioactive maloyl glucans from aloe vera gel: isolation, structure elucidation and in vitro bioassays. Carbohydr Res. 2006 Feb;341(3):355-64. http://dx.doi.org/10.1016/j.carres.2005.11.022. PMid:16343466.

25. Songsiripradubboon S, Kladkaew S, Trairatvorakul C, Sangvanich P, Soontornvipart K, Banlunara W, et al. Stimulation of dentin regeneration by using acemannan in teeth with lipopolysaccharide-induced pulp inflammation. J Endod. 2017 Jul;43(7):1097-103. http://dx.doi.org/10.1016/j. joen.2017.01.037. PMid:28477996.

\section{CONFLITOS DE INTERESSE}

Os autores declaram não haver conflitos de interesse.

\section{*AUTOR PARA CORRESPONDÊNCIA}

Larissa Cordeiro Cavalcante, Programa de Pós-graduação em Odontologia, UPFI - Universidade Federal do Piauí, Avenida Nossa Senhora de Fátima, Planalto Ininga, 64049-280 Teresina - PI, Brasil, e-mail: larissacordeirocavalcante@gmail.com 